\title{
Effect of local surgery on outcomes of stage IV breast cancer
}

\author{
Takahiro Tsukioki, Tadahiko Shien, Hiroyoshi Doihara \\ Department of Breast and Endocrine Surgery, Okayama University Hospital, Okayama, 700-8558, Japan \\ Contributions: (I) Conception and design: T Shien, T Tsukioki; (II) Administrative support: T Shien; (III) Provision of study materials or patients: \\ T Shien ; (IV) Collection and assembly of data: T Shien, T Tsukioki; (V) Data analysis and interpretation: T Shien, T Tsukioki; (VI) Manuscript \\ writing: All authors; (VII) Final approval of manuscript: All authors. \\ Correspondence to: Tadahiko Shien, MD, PhD. Department of Breast and Endocrine Surgery, Okayama University Hospital, 2-5-1 Shikata-cho, Kita- \\ ku, Okayama 7008558, Japan. Email: tshien@md.okayama-u.ac.jp.
}

\begin{abstract}
Metastatic breast cancer (MBC), including de novo stage IV, is regarded as being incurable and the mainstay of clinical management is systemic therapy. Traditionally, locoregional surgery is performed only for local control, such as to prevent ulceration and bleeding. In recent years, however, both retrospective and prospective studies have demonstrated the prognostic efficacy of primary surgery for de novo stage IV patients. Therefore, we conducted a meta-analysis to evaluate whether surgical therapy contributes to overall survival (OS) extension. We searched for clinical trials published in electronic databases (PubMed, Embase, and the Cochrane databases) and performed a meta-analysis of the data collected. There were five prospective randomized controlled phase III trials (RCTs). The results of three have been reported. According to our meta-analysis of these RCTs, primary surgery for de novo stage IV breast cancer patients significantly improves OS. However, the Tata trial showed that systemic therapy does not achieve a sufficient effect. Another trial, conducted in Turkey, had statistical shortcomings and patient randomization was not adequately performed The ABCSG (Austrian Breast and Colorectal Cancer Study Group) trial had too few subjects. Meta-analysis of 12 retrospective studies showed that patients with stage IV breast cancer receiving surgery as the initial treatment experienced longer OS (HR: 0.65, $\mathrm{P}<0.00001)$. Based on our meta-analysis of three reported RCTs, surgery as the primary treatment does not significantly impact the outcomes of de novo stage IV breast cancer patients. However, these trials had limitations. We await the results of the remaining two ongoing RCTs (ECOG 2108 and JCOG 1017). These trials are anticipated to resolve current controversies and provide many eagerly awaited answers.
\end{abstract}

Keywords: Breast cancer; metastatic; stage IV; local therapy; locoregional therapy; surgery; survival

Submitted Dec 23, 2019. Accepted for publication Jan 16, 2020.

doi: $10.21037 /$ tcr.2020.01.60

View this article at: http://dx.doi.org/10.21037/tcr.2020.01.60

\section{Introduction}

Breast cancer is one of the leading causes of cancer-related mortality in women worldwide. Significant progress has been made in the diagnosis and treatments of breast cancer, but the clinical outcomes of patients remain discouraging $(1,2)$. Despite improvements in diagnostic techniques and awareness of screening, patients with distant metastasis at diagnosis (de novo stage IV breast cancer) still have a poor prognosis. Reportedly, de novo stage IV disease accounts for approximately $10 \%$ of breast cancers $(3,4)$.
With the development of systemic therapies, the outcomes of breast cancer patients have been improving. Prolongation of overall survival (OS) has also been recognized in patients with metastatic breast cancer (MBC). In a French study conducted in 2004, which examined 724 patients with de novo MBC, $27 \%$ of patients during diagnosed during the period from 1987 to 1993 survived for 3 at least years, while the rate was $44 \%$ for those diagnosed between 1994 and 2000 (5). A more recent report from the USA, based on data from the Surveillance, Epidemiology and End Results (SEER) registries of the National Cancer 
Institute, described similar trends. The hazard ratio (HR) of OS was 0.81 in the groups diagnosed after 2007 (6). The mainstay of management for these patients is systemic therapies including chemotherapy, endocrine agents and tailored treatments targeting the biological features of their tumors (7). Local and metastatic sites respond very similarly to systemic therapy. Traditionally, aggressive surgery for the primary tumor is not recommended for de novo stage IV breast cancer due to lack of evidence supporting the efficacy of this approach in patients with advanced disease. However, local symptoms such as pain, bleeding, anemia, infection, and cachexia can significantly reduce quality of life (QOL) and primary surgery offers the possibility of ameliorating these local symptoms. Moreover, many retrospective studies have found that de novo stage IV breast cancer patients undergoing primary surgery may achieve better OS (8). Furthermore, worldwide, five prospective randomized studies have been launched with the goal of examining the efficacy of primary surgery for MBC. We conducted a metaanalysis of the results of these trials, to evaluate whether surgical therapy contributes to OS extension in patients with de novo stage IV breast cancer.

\section{Methods}

\section{Literature search and study selection}

We searched for clinical trials published in electronic databases (PubMed, Embase, and the Cochrane databases) through December 2018. The key words were "breast neoplasms", "mastectomy" and "the equivalent of stageIV breast cancer". In total, 465 potentially relevant reports were identified.

Our meta-analysis was conducted in accordance with the Preferred Reporting Items for Systematic Reviews and Meta-analyses (PRISMA) (9).

The following inclusive selection criteria were applied:

(I) Study population: MBC patients with no prior history of breast cancer diagnosis and who had not undergone primary surgery.

(II) Study design: studies examining the effect of local surgery in MBC patients were collected from available databases. Studies published as reviews, editorials, letters and comments were excluded.

(III) Outcome measures: OS reported for groups with versus without local surgery.

In the event of the same clinical case appearing in several publications, to avoid duplication of information, we included only the most informative article or that with the longest follow-up period.

\section{Statistical analysis}

Randomized controlled phase III trials (RCT) obtain a high level of evidence and the results have high clinical relevance. Thus, we conducted a quantitative and qualitative meta-analysis to evaluate the OS extension effect of primary surgical treatment. However, since not all RCT results are as yet available and the number of patients is small, we also evaluated the results of retrospective studies by performing a separate meta-analysis of RCTs. Both meta-analyses were performed using Review Manager 5.3.

\section{Results}

\section{Prospective trials}

We identified five RCTs designed to evaluate the prognostic efficacy of resecting the primary lesion in patients with de novo stage IV breast cancer. The five trials evaluated were the NCT 00193778 trial from India, the NCT 00557986 (MF07-01) trial from Turkey, the NCT 01242800 (ECOG 2108) trial from the USA, the NCT 01015625 (ABCSG-28 POSITIVE) trial from Austria and the UMIN 000005586 (JCOG 1017 PRIM-BC) trial from Japan (10-14).

All five trials have completed registration and three have reported the results obtained. All of these trials aimed to determine whether OS extension is achieved by primary surgery in patients with de novo stage IV breast cancer. There were some differences in the timing of surgery and types of systemic therapy administered. Three of the five trials examined meta-analyzed required initiation of systemic therapy prior to randomization, while others randomized the patients first, with versus without surgery, prior to systemic therapy. The designs of all five studies analyzed herein are summarized in Table S1.

The following results were obtained in the three RCTs reported to date.

\section{Indian trial (NCT 0019377)}

This was a single-institution RCT conducted at the Tata Memorial Cancer Institution in Mumbai. Patients were registered from 2005 to 2012. In this trial, the study protocol was that patients first received chemotherapy, consisting primarily of six cycles of anthracycline-based chemotherapy. Just $5 \%$ of patients also received a taxane. If a specific 
treatment effect was achieved, the patients were randomized into either the surgery or the non-surgery group. Initially, 716 patients were registered. Of the 716 patients, 440 showed a response to chemotherapy, but 90 of these were excluded for various reasons. Thus, the final count of patients eligible for randomization was 350 . They were randomized to either continue with systemic therapy alone or undergo primary local surgery with or without radiation (RT) plus continuation of systemic therapy. After randomization, 173 patients were assigned to receive primary local surgery and 177 continued systemic therapy alone. The primary endpoint was OS, and secondary endpoints were locoregional progression-free survival (PFS), distant PFS and healthrelated QOL.

At the median follow-up of 23 months, 235 had died. The median OS was 19.2 months in the surgical group, 20.5 months in the non-surgical group, not a statistically significant difference ( $\mathrm{HR}=1.04 . \mathrm{P}=0.79$ ) Based on the secondary endpoint results, locoregional PFS was significantly better in the surgical group $(\mathrm{HR}=0.16$. $\mathrm{P}=0.001$ ), while distant PFS was significantly better in the systemic therapy only group $(\mathrm{HR}=1.42 . \mathrm{P}=0.012)$.

\section{MF07-01 trial (NCT 00557986)}

Patients were registered from 2008 to 2012. Unlike the India trial, the protocol of this trial was to first randomize the patients into surgery versus non-surgery groups and then compare the prognostic effects of primary local surgery. Initially, 312 patients were registered, and 274 were ultimately evaluable and included in the study. After randomization, 138 patients were assigned to receive primary local surgery with or without RT. In addition, the local surgery required free margins in all cases undergoing resection and included axillary dissection if axillary lymph node metastasis was detected. The systemic therapy regimen, in both groups, was for all patients with hormone receptorpositive $\mathrm{MBC}$ to receive endocrine therapy and all with human epidermal growth factor receptor 2 (HER2) positive cancer to receive trastuzumab, with a chemotherapy regimen consisting primarily of anthracycline-based therapy. The primary endpoint was three-year survival, and the OS difference between the two groups was $18 \%$ (35\% in the surgical and $17 \%$ in the non-surgical group). Secondary endpoints were morbidity, locoregional PFS and QOL.

There was no significant difference in three-year survival, the primary endpoint, between the two groups. However, the post-analysis evaluation revealed a tendency for OS extension, with a median OS of 46 months in the surgical group and 37 months in non-surgical group (HR $=0.66, \mathrm{P}=0.005)$. The secondary endpoint of locoregional PFS was $1 \%$ in the surgery group and $11 \%$ in the nonsurgical group, showing a statistically significant difference $(\mathrm{P}=0.001)$.

\section{ABCSG-28 POSITIVE trial (NCT 01015625)}

This trial registered patients from 2010 to 2019. The protocol was similar to that of the Turkish trial. Patients were first randomized to locoregional therapy or systematic therapy. Patients in the surgical group received primary breast and axillary surgery, with or without RT. After these locoregional therapies, systemic therapy was initiated. However, even in the non-surgical group, surgery was allowed when deemed necessary. The primary endpoint was OS, and the secondary endpoints were locoregional PFS and distant PFS. The planned number of registrations was 516 , but the trial was terminated after 90 cases had been registered, 45 per arm, and the results were then assessed and reported.

The median OS was 34.6 months in the surgical group, 54.8 months in the non-surgical group, not a statistically significant difference $(\mathrm{HR}=0.691, \mathrm{P}=0.267)$.

We summarized the results of the three reported RTCs examining the effects of surgery on OS. The NCT 0019377 trial, which registered 350 patients and had a primary endpoint of OS, showed no significant difference with versus without surgery. The median OS was 19.2 months in the surgical and 20.5 months in the non-surgical group ( $\mathrm{HR}=1.04$. $\mathrm{P}=0.79$ ). In the ABCSG-28 trial, which included 90 patients, a tendency for worsening of OS was noted with surgery, but the difference did not reach statistical significance. The median OS was 34.6 months in the surgical and 54.8 months in the non-surgical group. On the other hand, in the MF07-01 trial, 274 patients were registered, and a tendency for OS to be extended was observed, with median OS being 46 months in the surgical and 37 months in the non-surgical group (HR $=0.66, \mathrm{P}=0.005$ ) (Table S2). According to our meta-analysis of these three RCTs, surgical treatment of MBC does not significantly prolong OS (Figure 1).

The following two trials have completed patient enrollment and the results are eagerly anticipated.

\section{ECOG2108 trial (NCT 0124288)}

Patients were registered from 2011 to 2015. This protocol was for patients diagnosed as having de novo stage IV breast cancer to first be given systemic therapy. After 


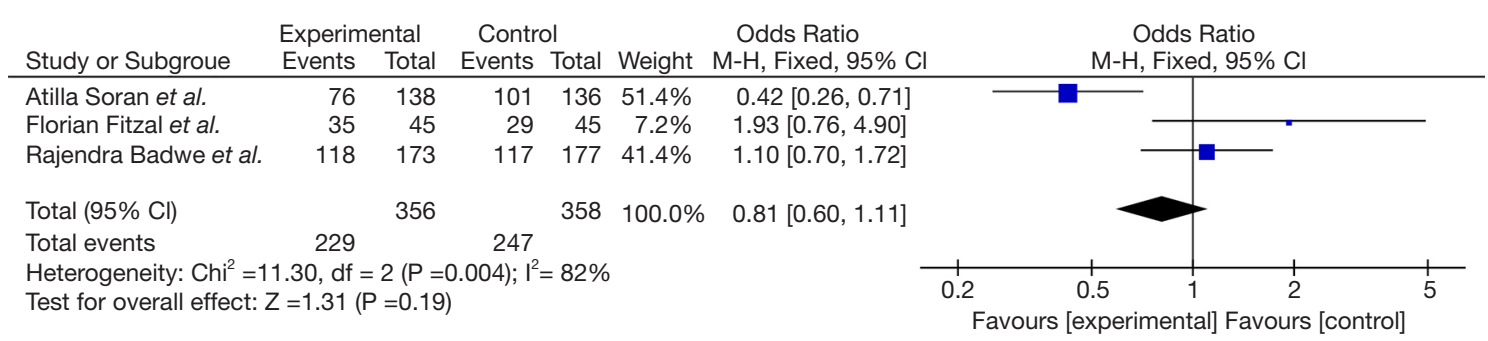

Figure 1 Results of a meta-analysis of 3 prospective studies examining the effect of surgical treatment on OS in patients with metastatic breast cancer.

4 to 8 months of systemic therapy, patients showing treatment effects, such as stable or responsive disease, were randomized into surgery and non-surgery groups. The initial systemic therapies included endocrine agents, chemotherapy and anti-HER2 agents. The surgical group received surgery and RT, the standard protocol for local disease in a non-metastatic setting. The planned number of patients was 880, but registration was closed in 2015 with only 383 patients. The primary endpoint was OS at three years, and the study was redesigned with power to detect a $19 \%$ difference between the surgical and non-surgical groups. The secondary endpoints were local PFS and QOL.

\section{JCOG 1017 PRIM-BC trial (UMIN 000005586)}

Patients were registered from 2011 to 2018. As in the USA and India trials, patients initially received systemic therapy according to tumor biology for three months. Subsequently, those patients in whom treatment achieved an effect were randomized into surgery and non-surgery groups. The surgical procedures were limited to local tumor resection, with neither axillary dissection nor RT. The primary endpoint was OS and the secondary endpoints were distant PFS, rate of uncontrolled local disease and complications from surgery. The planned number of patients was 410 and registration was completed when this number was reached. The results will be reported in 2022 .

\section{Retrospective trials}

By performing a literature search according to PRISMA, we identified 12 retrospective clinical trials focusing on whether or not to remove the primary lesion in patients with de novo stage IV breast cancer (15-17). In total, 28,400 patients were included in these trials and 14,905 had received local surgery. There were variations in the methods and timing of surgery among the trials. Axillary management, especially, was more difficult to evaluate based on the analysis of retrospective studies which do not consistently obtain detailed information on this feature of treatment.

Our meta-analysis of the 12 retrospective reports revealed that primary surgery for patients with stage IV breast cancer prolonged OS (HR: 0.65, $\mathrm{P}<0.00001$ ) (Figure 2).

\section{Discussion}

Our meta-analysis of prospective studies, RCTs, yielded results opposite those of retrospective studies. From the viewpoint of the strength of evidence, the RCT results merit greater emphasis. The retrospective trials have several inherent biases, including those reflecting patient age, the timing of surgery, the status of cancer metastasis, the eligibility criteria applied and so on. These trials could not be analyzed in detail or with sufficient accuracy. There is a high possibility that the MBC patients for whom doctors deemed surgery to be necessarily had distant metastases, with surgery having a good local effect on symptoms while not impacting OS. In addition, in retrospective studies especially, the applications of RT varied markedly. The effect of locoregional RT for MBC was demonstrated in two studies $(18,19)$. Le Scodan et al. reported that MBC patients who received locoregional RT alone showed significantly better OS than those who did not (HR =0.7). Nguyen $e$ al . reported that there was a significant five-year OS advantage in those undergoing locoregional RT as compared to the group without RT $(\mathrm{P}<0.001)$. RT might also contribute to better OS.

However, the three reported RCTs had limitations, such that the results must be interpreted with caution.

First, the timing of breast surgery differed among the trials. In the Indian trial (NCT 00193778), the patients received prescribed systemic therapy and were subsequently randomized to the surgery and non-surgery groups. On the other hand, in the other two trials (ABCSG-28, MF0701 ), the patients were initially divided into surgery or non- 


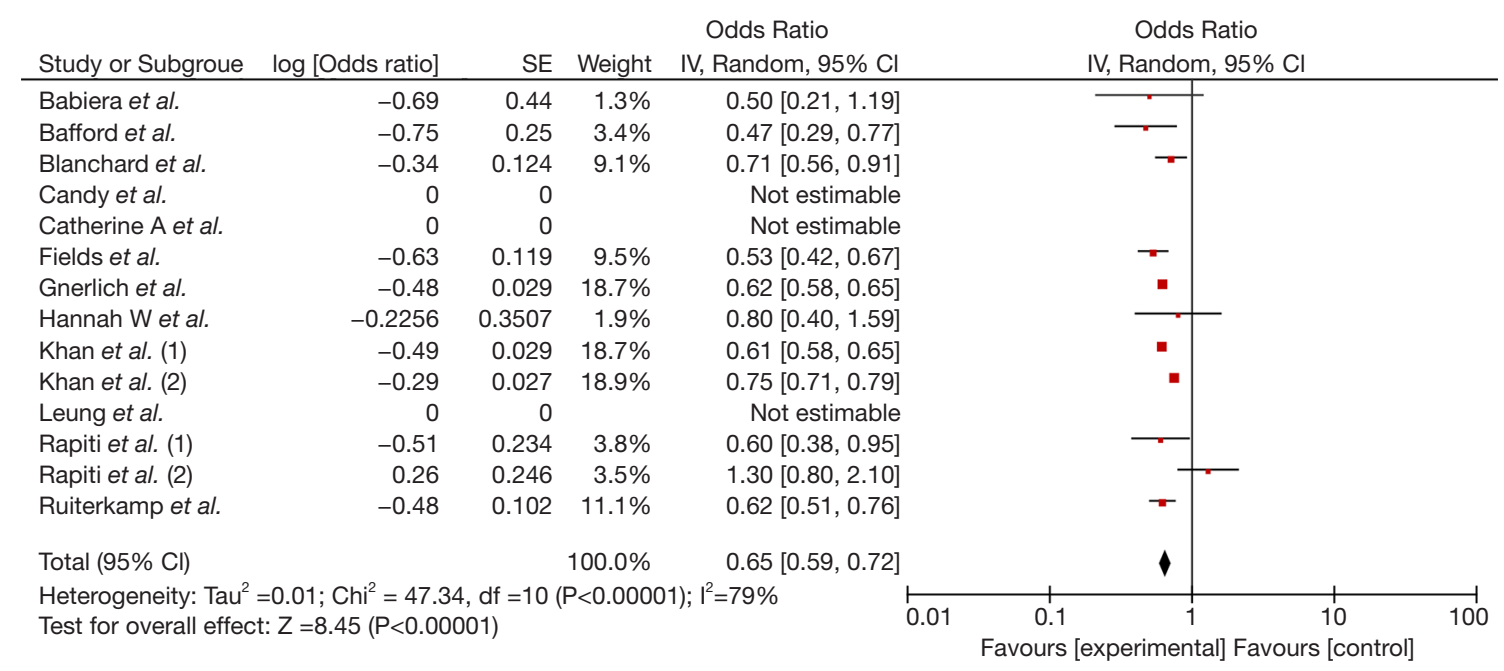

Figure 2 Results of a meta-analysis of 12 retrospective studies examining the effects of surgical treatment on OS in patients with metastatic breast cancer.

surgery groups and then received systemic therapies.

Second, in the trial from India (NCT 00193778 trial), there were very few systemic treatments after randomization as compared to industrialized nations. Furthermore, there was a lack of standard systemic treatments tailored to cancer biology, such as hormonal, chemotherapeutic and targeting agents (e.g., trastuzumab). Consequently, the PFS and OS in this trial were shorter than in the other two trials. The short PFS in the surgical group after randomization may have been due to the lack of effective systemic treatment.

Third, on the MF07-01 trial (NCT 00557986), the randomization assignment factors may not have been appropriate. For this reason, patients with triple-negative type breast cancer, which is considered to have a poor prognosis, were more numerous in the non-surgery group. Moreover, the originally configured endpoint might not have been appropriate, since the overall survival rate was the originally planned endpoint, rather than OS itself. The method applied for diagnosing metastasis may also have produced ambiguous results as established guidelines were lacking.

Fourth, in the trial from Europe (ABCSG-28 trial), the number of participants was considerably less than planned. The planned number was 516 but only 90 patients were actually enrolled. As such, inadequate statistical power may account for the lack of a significant difference.

For these reasons, patient backgrounds vary markedly among these RCTs. This meta-analysis detected no effect of surgery on the OS of breast cancer patients with stage IV disease. We eagerly await the results of the two remaining RCTs (JCOG1017 and ECOG2108 trial).

\section{Conclusions}

Our meta-analysis of the three RCTs reported to date, detected no significant of effect of primary surgery on the OS of patients with de novo stage IV breast cancer. Unfortunately, data on the effects of early surgery for the prevention of local symptoms are as yet insufficient. We must always carefully weigh the risks and benefits of surgical treatments. We await the results of the two-remaining ongoing RCTs (ECOG 2108 and JCOG 1017). These trials are anticipated to resolve current controversies and provide information useful for managing MBC patients.

\section{Acknowledgments}

Funding: This report was supported in part by the Practical Research for Innovative Cancer Control (19ck0106307h0002) from the Japan Agency for Medical Research and Development, AMED.

\section{Footnote}

Provenance and Peer Review: This article was commissioned by the Guest Editors (Tadahiko Shien and Kaori Terata) for the series "Loco regional therapy for metastatic breast cancer" published in Translational Cancer Research. The 
article was sent for external peer review organized by the Guest Editors and the editorial office.

Conflicts of Interest: All authors have completed the ICMJE uniform disclosure form (available at http://dx.doi. org/10.21037/tcr.2020.01.60). The series "Loco-regional therapy for metastatic breast cancer" was commissioned by the editorial office without any funding or sponsorship. The authors have no other conflicts of interest to declare.

Ethical Statement: The authors are accountable for all aspects of the work in ensuring that questions related to the accuracy or integrity of any part of the work are appropriately investigated and resolved.

Open Access Statement: This is an Open Access article distributed in accordance with the Creative Commons Attribution-NonCommercial-NoDerivs 4.0 International License (CC BY-NC-ND 4.0), which permits the noncommercial replication and distribution of the article with the strict proviso that no changes or edits are made and the original work is properly cited (including links to both the formal publication through the relevant DOI and the license). See: https://creativecommons.org/licenses/by-nc-nd/4.0/.

\section{References}

1. Perou CM, Sorlie T, Eisen MB, et al. Molecular portraits of human breast tumours. Nature 2000;406:747-52.

2. Sotiriou C, Pusztai L. Gene-expression signatures in breast cancer. N Engl J Med 2009;360:790-800.

3. Sant M, Allemani C, Berrino F, et al. Breast carcinoma survival in Europe and the United States. Cancer 2004;100:715-22.

4. Siegel R, Naishadham K, Bachelot T, et al. Cancer statistics, 2013. CA Cancer J Clin 2013;63:11-30.

5. Andre F, Slimane K, Bachelot T, et al. Breast cancer with synchronous metastases: trends in survival during a 14year period. J Clin Oncol 2004;22:3302-8.

6. Thomas A, Khan SA, Chrischilles EA, et al. Initial surgery and survival in stage IV breast cancer in the United States, 1988-2011. JAMA Surg 2016;151:424-31.

7. Gradishar WJ, Anderson BO, Balassanian R, et al. Breast cancer version 2. 2015. J Natl Compr Canc Netw 2015;13:448-75.

8. Khan SA. Dose resection of an intact breast primary improve survival in metastatic breast cancer? Oncology 2007;21:924-31.
9. Moher D, Liberati A, Tetzlaff J, et al. Preferred reporting items for systematic reviews and meta-analyses: the PRISMA statement. PLoS Med 2009;6:e1000097.

10. Badwe R, Hawaldar R, Nair N, et al. Locoregional treatment versus no treatment of the primary tumour in metastatic breast cancer: an open-label randomised controlled trial. Lancet Oncol 2015;16:1380-8.

11. Soran A, Ozmen V, Ozbas S, et al. Randomized Trial Comparing Resection of Primary Tumor with No Surgery in Stage IV Breast Cancer at Presentation: Protocol MF07-01. Ann Surg Oncol 2018;25:3141-9.

12. Fitzal F, Bjelic-Radisic V, Knauer M, et al. Impact of Breast Surgery in Primary Metastasized Breast Cancer: Outcomes of the Prospective Randomized Phase III ABCSG-28 POSYTIVE Trial. Ann Surg 2019;269:1163-9.

13. Shien T, Nakamura K, Shibata T, et al. A randomized controlled trial comparing primary tumour resection plus systemic therapy with systemic therapy alone in metastatic breast cancer (PRIM-BC): Japan Clinical Oncology Group Study JCOG1017. Jpn J Clin Oncol 2012;42:970-3.

14. Khan SA, DesJardin ESM. Readdressing the Role of Surgery of the Primary Tumor in de Novo Stage IV Breast Cancer. Cancer Treat Res 2018;173:73-88.

15. Ruiterkamp J, Voogd AC, Bosscha K, et al. Impact of breast surgery on survival in patients with distant metastases at initial presentation: a systematic review of the literature. Breast Cancer Res Treat 2010;120:9-16.

16. Akay CL, Ueno NT, Chisholm GB, et al. Primary tumor resection as a component of multimodality treatment may improve local control and survival in patients with stage IV inflammatory breast cancer. Cancer 2014;120:1319-28.

17. Hazard HW, Gorla SR, Scholtens D, et al. Surgical resection of the primary tumor, chest wall control, and survival in women with metastatic breast cancer. Cancer 2008;113:2011-9.

18. Le Scodan R, Stevens D, Brain E, et al. Breast cancer with synchronous metastases: survival impact of exclusive locoregional radiotherapy. J Clin Oncol 2009;27:1375-81.

19. Nguyen DH, Truong PT, Alexander C, et al. Can locoregional treatment of the primary tumor improve outcomes for women with stage IV breast cancer at diagnosis? Int J Radiat Oncol Biol Phys 2012;84:39-45.

Cite this article as: Tsukioki T, Shien T, Doihara H. Effect of local surgery on outcomes of stage IV breast cancer. Transl Cancer Res 2020;9(8):5102-5107. doi: 10.21037/tcr.2020.01.60 
Table S1 Summary of the protocols of five prospective randomized trials

\begin{tabular}{|c|c|c|c|c|c|c|c|c|}
\hline Trial group & Trial number & Accrual period & $\begin{array}{l}\text { Accrual number } \\
\text { of patients }\end{array}$ & Target cases & Protocol & & $\begin{array}{l}\text { Primary } \\
\text { outcome }\end{array}$ & Secondary outcome \\
\hline India & NTC00193778 & $2005-2013$ & $716(350)$ & De novo Stage IV & $\begin{array}{l}\text { Systemic chemotherapy ( } 6-8 \text { cycles) } \rightarrow \text { objective response } \rightarrow \\
\text { randomized locoregional treatment or not } \rightarrow \text { only luminal type } \\
\text { received endcrine therapy }\end{array}$ & $\mathrm{Bp} / \mathrm{BT}+\mathrm{Ax} \pm \mathrm{RT}$ & os & LPFS, DPFS, QOL \\
\hline Turkey & $\begin{array}{l}\text { NCT00557986 } \\
\text { (MF07-01) }\end{array}$ & 2007-2012 & 274 & De novo Stage IV & Randomized locoregional therapy or not $\rightarrow$ systemic therapy & $\mathrm{Bp} / \mathrm{BT}+\mathrm{Ax} \pm \mathrm{RT}$ & os & LPFS, morbidity, QOL \\
\hline Austria & $\begin{array}{l}\text { NCT01015625 } \\
\text { (ABCSG 28) }\end{array}$ & 2011-2015 & 90 & De novo Stage IV & Randomized locoregional therapy or not $\rightarrow$ systemic therapy & $\mathrm{Bp} / \mathrm{BT}+\mathrm{SNB}(\rightarrow \mathrm{Ax}) \pm \mathrm{RT}$ & os & LPFS, DPFS \\
\hline USA & $\begin{array}{l}\text { NCT01242800 } \\
\text { (ECOG2108) }\end{array}$ & 2011-2015 & $368(258)$ & De novo Stage IV & $\begin{array}{l}\text { Systemic therapy ( } 4-8 \text { months) } \rightarrow \text { objective response } \rightarrow \text { randomized } \\
\text { locoregional treatment or not } \rightarrow \text { continue systemic therapy }\end{array}$ & $\mathrm{Bp} / \mathrm{BT}+\mathrm{SNB}(\rightarrow \mathrm{Ax}) \pm \mathrm{RT}$ & os & LPFS, QOL \\
\hline JAPAN & $\begin{array}{l}\text { UMIN000005586 } \\
\text { (JCOG1017) }\end{array}$ & $2011-2018$ & $570(407)$ & De novo Stage IV & $\begin{array}{l}\text { Systemic therapy }(2 \text { months }) \rightarrow \text { objective response } \rightarrow \text { randomized } \\
\text { locoregional treatment or not } \rightarrow \text { continue systemic therapy }\end{array}$ & $\mathrm{Bp} / \mathrm{BT}$ & OS & $\begin{array}{l}\text { LPFS, DPFS, proportion of } \\
\text { uncontrolled local tumor }\end{array}$ \\
\hline
\end{tabular}

Table S2 Results of the three reported prospective trials

\begin{tabular}{|c|c|c|c|c|c|c|c|c|c|c|c|c|c|}
\hline \multirow{2}{*}{ Study } & \multirow{2}{*}{$\begin{array}{l}\text { Randomized } \\
\text { number }\end{array}$} & \multicolumn{2}{|c|}{ The number of patients } & \multirow{2}{*}{$\begin{array}{l}\text { Median observation } \\
\text { period }\end{array}$} & \multicolumn{3}{|c|}{ OS } & \multicolumn{3}{|c|}{ Distant PFS } & \multicolumn{3}{|c|}{ Local PFS } \\
\hline & & Surgery & Non-surgery & & Non-surgery & Surgery & HR (P value) & Non-surgery & Surgery & HR ( $P$ value) & non-surgery & Surgery & HR (P value) \\
\hline India & 350 & 173 & 177 & 23 & 19.2 & 20.5 & $1.04(\mathrm{P}=0.79)$ & 11.3 & 19.8 & $1.42(P=0.012)$ & NA & 18.2 & $0.16(P<0.0001)$ \\
\hline Turkey & 274 & 138 & 136 & & 46 & 37 & $0.66(P=0.005)$ & & & & & & \\
\hline Austria & 90 & 45 & 45 & 37.5 & 34.6 & 54.8 & $0.69(P=0.267)$ & 13.9 & 29 & $0.598(P=0.067)$ & & & \\
\hline
\end{tabular}

